\title{
MS09-03 | Vagabond: Redefining the Model for Macromolecular Refinement
}

Ginn, Helen (Diamond Light Source Ltd, Didcot, GBR)

Model refinement for biomolecular crystallography, at present, relies on a model defined in atomic $x, y, z$ parameters and associated B factors. Vagabond is a new refinement project which takes an unconventional approach, revisiting the concept of refining in torsion space by defining the model in terms of bond lengths, angles and torsion angles. B factors have been replaced by defining the flexibility of the protein through allowing variation in the torsion angles. The distribution of the torsion angles within this model can then be sampled multiple times, creating an ensemble of individual structures: the sum of this ensemble is equivalent to the estimation of electron density. This significantly reduces the number of parameters to describe the structure and is capable of describing atomic distributions which are not accessible from isotropic or anisotropic B factor models alone. The combination of describing a more complex distribution with fewer parameters leads to a reduction in overfitting and increased clarity of maps as developed against existing structures from X-ray crystallography data. This reveals verifiable electron density differences in the reported structures from the Protein Data Bank, particularly revealing features in low resolution structures which, formerly, could only be obtained by optimisation of crystal conditions and collecting a new high resolution data set. Although the improvement in electron density quality is conspicuous across all resolution ranges, the parameter reduction is particularly powerful at low resolution, where fewer observations are available to support the model parameterisation. 